\title{
Screening for fetal abnormalities: From a health technology assessment report to a national statute
}

\author{
Ilona Autti-Rämö \\ Finnish Office for Health Care Technology Assessment (Finohta), STAKES \\ Marjukka Mäkelä \\ Finnish Office for Health Care Technology Assessment (Finohta), STAKES and University \\ of Copenhagen
}

Objectives: Previously in Finland, each municipality selected which methods to use in screening for fetal abnormalities. This resulted in practice variation and inequity. The national health technology assessment (HTA) office Finohta compared the methods and time frames available when screening for chromosomal and structural abnormalities. The assessment identified a need to discuss several value-laden questions before policy decisions could be taken.

Methods: The National Screening Committee (NSC) at the Ministry of Health and Social Affairs $(\mathrm{MOH})$ formulated a statement based on the HTA report to inform policy makers. This article describes the steps in the policy process.

Results: The national screening committee organized an open forum to discuss the ethically relevant aspects of fetal screening, and a lengthy public discussion highlighted the viewpoints of various stakeholders. Based on the assessment, public discussion, and several committee meetings that heard further experts, the NSC formulated a conclusion based on equity of access. This also offered options for families unwilling to terminate a pregnancy due to fetal abnormality. The $\mathrm{MOH}$ sent the conclusion to a comment round, and the proposal was accepted with minor adjustments. The Ministry decided to unify the regulation of all public screening programs by one statute. The policy process lasted three years, and the methods to screen for fetal abnormalities will be unified after a further 3 years.

Conclusions: The assessment of screening programs for fetal abnormalities reached beyond its original mandate. The process of examining values behind screening resulted in a program that respects the differing objectives and ethical values of pregnant families, and to national legislation on screening programs.

Keywords: Screening, Health policy, Prenatal, Pregnancy, Fetus, Chromosomal abnormalities, Malformation

Major congenital abnormalities are detected in 2 to 3 births per 100, and 1 per 100 is affected by several major anomalies

We thank the members of the HTA project on prenatal screening. We also thank the members of the national Screening Committee for their commitment to reach a statement that would ensure equity of care for all pregnant women. or a syndrome $(5 ; 6$; Finnish Registry of Congenital Malformations). The most severe anomalies can lead to perinatal death or permanent disability. Some disorders can be treated during the fetal period or require immediate treatment after birth to reduce permanent disability or prevent the death of the newborn. 
The methods to screen for chromosomal abnormalities, especially trisomy 21 (Down syndrome) have been in use since the 1980s (7) and are still developing (12). Ultrasound techniques are increasingly used to screen for structural abnormalities of the fetus. The chances of detecting structural abnormalities vary depending on the type and severity of the anomaly, the scanning equipment, the experience of the sonographer and the timing of screening $(5 ; 9 ; 10)$. The existing variations and difficulties in determining when a malformation is severe, moderate, or mild raises major ethical questions every time a malformation is identified during the fetal period.

Between and within countries there is considerable variation in the use of various existing screening methods. In Finland, each of the over 400 municipalities can independently decide which screening method to provide, resulting in a wide variety in practices, inequity, and major problems in counseling and organizing the investigations. According to a survey in 2002, forty of the forty-four hospitals with a maternity clinic offered a nuchal translucency measurement for chromosomal screening but only four of them combined it with serum markers (11). Forty hospitals offered age-based direct invasive diagnostic procedures (chorionic villi sample or amniocentesis) to detect chromosomal anomalies. Of these, four had set the age limit at 35 , one at 36 , seventeen at 37 , three at 38 , three at 39 , and twelve at 40 years. In Finland, ultrasound examination has only been offered as part of screening (measurement of nuchal translucency or screening for structural malformations) or due to clinical symptoms.

The Finnish organization for gynecologists and the local health decision makers requested a health technology assessment (HTA) on the topic, while the Ministry of Health and Social Affairs also needed this information to be used as a basis for policy decisions. In this article, we describe the process from initial request to a national statute on screening. The questions identified by the HTA assessment, the process of answering these questions and the selection of screening methods to be offered for pregnant women in Finland are presented.

\section{ASSESSMENT OF OPTIONS FOR SCREENING FOR STRUCTURAL AND CHROMOSOMAL ABNORMALITIES}

The HTA assessment was conducted together with methodological experts on HTA (IAR, MM) and health economics, the head of the national registry for anomalies, and a panel of clinical experts in obstetrics, neonatology, general practice, and clinical genetics. In addition to being methodological experts, the authors of this article are also clinical experts in pediatric neurology (I.A.R.) and general practice (M.M.).

The possible screening models for Finland were constructed using evidence from the literature and the clinical experts (1). Optimal methods and time frames to be used when screening for chromosomal abnormalities were identified in a recent HTA report (12). For the chromosomal abnormalities, the following options were considered to be applicable in Finland. (i) The combination of serum markers (PAPP-A and $\beta$-HCG at gestation week 8 to 10 ) and measurement of nuchal translucency (ultrasound at gestation week 10 to 12), (ii) triple serum markers (estriol, $\beta$-HCG, AFP at gestation week 14 to 15 ), or (iii) a combination of $\mathrm{i}$ and ii. For structural abnormalities, the options were based on recent literature on the detection rate during different gestation weeks $(5 ; 8-10)$, on Finnish legislation on the termination of pregnancy, and on equity in care. The options were (i) ultrasound screening during gestation week 18 to 21 and (ii) ultrasound screening after gestation week 24 for parents to whom termination of pregnancy would not be an option. In Finland, induced abortion due to fetal abnormalities is legal until the end of the 24th gestation week.

The costs of the various screening methods were calculated in euros at the 2004 price level. The effects and costs of various screening options were compared against a noscreening situation with the assumption that 90 percent of the pregnant women would participate. Sensitivity analyses were done for screening costs based on different participation rates. An example of the comparison of both chromosomal and structural screening against no screening situation is presented in Table 1.

The expert group also identified the ethical consequences of each screening model. The HTA draft was sent to external experts for peer review before publishing and their comments were included in the ethical evaluation. A summary of the ethical evaluation has been previously published (2).

Although the expert group could identify the best possible screening methods, several critical questions needed answering before a national decision could be taken on a uniform screening system. Questions regarding the aims of the screening programs are presented in Table 2. The expert group further required that the decision has to provide equity within the country. In addition, the quality of the screening program needs to be ensured (e.g., improved counseling at all levels of the screening program, criteria for training the sonographs, the need for centralization, requirements for the ultrasound equipment, setting the sensitivity and specificity levels, follow-up of induced harm).

\section{THE DECISION PROCESS IN THE NATIONAL SCREENING COMMITTEE}

The Screening committee at the Finnish Ministry of Social Welfare and Health was ready in October 2003 to evaluate the existing and proposed screening programs in Finland. Both authors of this article are members of the Screening Committee and had, thus, the opportunity to inform the Screening Committee during the HTA project, present the report, and answer content-specific questions. The Screening Committee 
recognized that the goal of the prenatal screening programs is to allow parents to take informed decision about the pregnancy. These decisions range from termination of pregnancy to optimal care during pregnancy and delivery. It was considered important to discuss the list of questions (examples in Table 2) in the HTA report with all stakeholders and an open seminar was organized.

Four hundred persons, from laypersons to health decision makers, participated in the seminar in October 2005. The seminar started with a computerized survey of the participant's opinions. The participants answered each question through remote control, and the results were shown immediately. The seminar included lectures and a panel discussion also involving patient organizations and layperson. The survey questions were repeated at the end of the seminar. The information received during the seminar had a clear impact on the opinions of the participants (Table 3). The seminar revealed new issues for the Screening Committee to tackle before issuing a final statement.

The Screening Committee invited the principal investigator at the national registry for fetal abnormalities to discuss the various scenarios following the options of each screening program. Finally, after six meetings and close to 1 year of hard work, the Screening Committee unanimously recommended a national screening program (the content explained later) that would ensure equity in care by providing the best currently available screening options for the citizens. Furthermore, the Screening Committee recommended that the effects and quality of the screening programs must be continuously monitored and quality ensured by a national group of experts. This group will determine the requirements for equipment and personnel in the program. The personnel in primary care (maternal health clinics) and secondary and tertiary hospitals needs training to give adequate and objective information and counseling.

\section{THE PROCESS AT THE MINISTRY OF HEALTH AND SOCIAL AFFAIRS}

The Ministry of Health and Social Affairs accepted the statement of the Screening committee and sent it for a wide round of commenting, including health decision makers, content experts, ethicists, and patient organizations. A total of fortyseven comments were given. The statement was well received, with only minor clarifications required. The ministry had further asked whether the uniform screening program should be implemented through a recommendation, a code of practice or a statute. Twenty of the respondents favored compulsory implementation by means of a statute so as to reach equity in care, whereas seven favored a recommendation and the rest were undecided.

After weighing the different options, the Ministry decided to regulate the screening programs for fetal abnormalities through a statute. As this strategy would increase the cost burden for communities, the ministry requested an estimate 
Table 2. Critical Questions on the Aim of Prenatal Screening

\section{Screening for chromosomal abnormalities}

Is it justified to screen for an abnormality that cannot be treated and leads to miscarriage or perinatal death (e.g., trisomies 13 and 18)?

Is it justified to screen for an abnormality that cannot be treated and leads often to miscarriage but can also lead to perinatal death, birth of a child with several other malformations, or birth of a child with only mild mental retardation (e.g., trisomy 21)?

If it is considered justified to screen for these abnormalities, how large should the risk of chromosomal abnormality be so that screening and invasive investigations are justified against the risk of miscarriage due to invasive diagnostic procedures?

If screening is offered only to older pregnant women, what would be the age limit?

If screening for chromosomal abnormalities is not publicly funded, what actions need to be taken in regards to offering screening in private clinics?

\section{Screening for structural malformations}

Is it justified to screen for an abnormality that cannot be treated and leads to miscarriage or perinatal death (e.g., anencephaly)?

Is it justified to screen for an abnormality that cannot be treated and can lead to a handicap of wide severity, ranging from total independence to continuous need for help (e.g., spina bifida)?

Is it justified to screen for an abnormality for which the outcome can be improved if care in pregnancy, in delivery, and for the newborn can be planned optimally (e.g., heart malformations)?

If the aim is on the one hand to allow termination of pregnancy in case of identified fetal abnormality but also to provide optimal care in pregnancy, in delivery, and for the newborn, when should the ultrasound be offered?

If the aim is to identify only abnormalities for which a treatment that improves the prognosis exists, when is the optimal timing for ultrasound scan?

If screening for structural abnormalities is not publicly funded, what actions need to be taken with regard to offering screening in private clinics?

Table 3. Survey of Opinions on Various Ethically Relevant Issues within the Context of Various Screening Options

\begin{tabular}{|c|c|c|c|c|}
\hline \multirow[b]{2}{*}{ Question $^{\mathrm{a}}$} & \multicolumn{2}{|c|}{$\begin{array}{l}\text { At the beginning } \\
\text { of the seminar }\end{array}$} & \multicolumn{2}{|c|}{$\begin{array}{l}\text { At the end of the } \\
\text { seminar }\end{array}$} \\
\hline & Yes $(\%)$ & No $(\%)$ & Yes $(\%)$ & No $(\%)$ \\
\hline $\begin{array}{l}\text { Is it justified to screen for an abnormality that cannot be treated and leads to miscarriage or } \\
\text { perinatal death (e.g., trisomies } 13 \text { and 18)? }\end{array}$ & 65 & 31 & 76 & 21 \\
\hline $\begin{array}{l}\text { Is it justified to screen for an abnormality that cannot be treated and leads often to miscarriage or } \\
\text { perinatal death, but the person can also have fairly good quality of life up to adulthood (e.g., } \\
\text { trisomy 21)? }\end{array}$ & 75 & 20 & 68 & 31 \\
\hline $\begin{array}{l}\text { Is it justified to screen for a structural abnormality that cannot be treated and the severity of the } \\
\text { handicap can vary from severe to mild (e.g., spina bifida)? }\end{array}$ & 83 & 14 & 86 & 13 \\
\hline $\begin{array}{l}\text { Is it justified to screen for a structural abnormality that can be treated and thus the prognosis can } \\
\text { be improved (e.g., heart malformation)? }\end{array}$ & 92 & 6 & 94 & 5 \\
\hline \multirow{2}{*}{\multicolumn{5}{|c|}{$\begin{array}{l}\text { Invasive diagnostic procedure in chromosomal screening can lead to miscarriage in about } 1 / 100 \text {. } \\
\text { What is the acceptable percentage of participants that are offered invasive diagnostic } \\
\text { procedures? }\end{array}$}} \\
\hline & & & & \\
\hline $1 \%$ & 14 & & 11 & \\
\hline $3 \%$ & 19 & & 39 & \\
\hline $5 \%$ & 20 & & 29 & \\
\hline $10 \%$ & 8 & & 3 & \\
\hline $\begin{array}{l}\text { Should the family themselves be allowed to choose the method to screen for chromosomal } \\
\text { abnormalities? }\end{array}$ & 67 & 26 & 54 & 37 \\
\hline Should the family be allowed to choose the timing for structural ultrasound scan? & 43 & 50 & 59 & 38 \\
\hline \multicolumn{5}{|l|}{ Should the structural ultrasound screening be performed } \\
\hline In University hospitals only & 9 & & 10 & \\
\hline In University and Central hospitals only & 43 & & 65 & \\
\hline In all maternity clinics & 25 & & 18 & \\
\hline Only in one center in Finland & 10 & & 2 & \\
\hline \multicolumn{5}{|l|}{ Should Finland offer screening for chromosomal and structural abnormalities with } \\
\hline A nationally uniform screening program & 75 & & 98 & \\
\hline A uniform screening program within a University hospital district & 12 & & 2 & \\
\hline A uniform screening program within a hospital district & 7 & & 0 & \\
\hline A program according to the wishes of each municipality & 3 & & 0 & \\
\hline
\end{tabular}

Note. Only "yes" and "no" answers are presented in the table, percentage of "don't know" answers can be counted when adding up to $100 \%$.

answers are given in parentheses. 


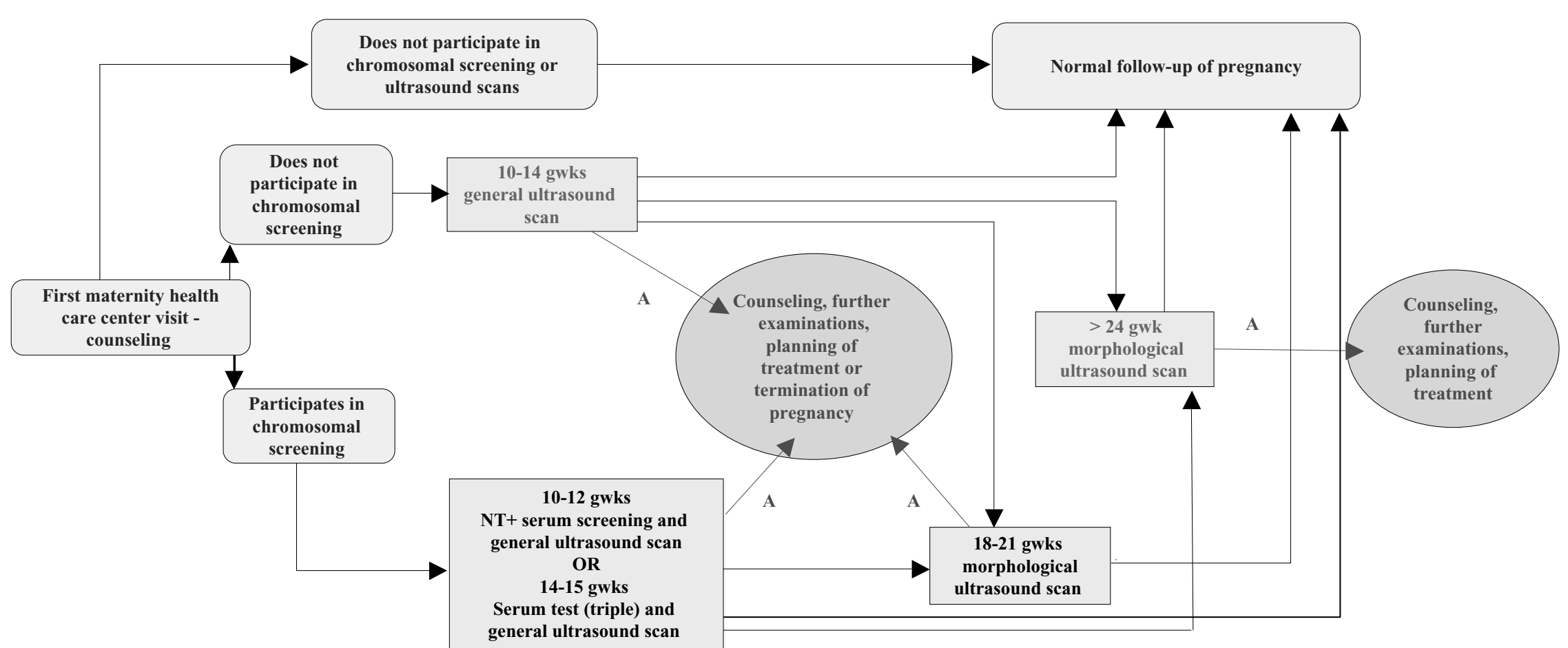

A= abnormal fetal finding

NT= nuchal translucency

gwks $=$ gestational weeks

Figure 1. The various pathways when deciding on participation. 
on the cost-impact of the various screening options on the municipalities. This estimate was not possible within the given time frames, as the Finohta report on fetal abnormalities had not looked at the costs of all program options recommended by the Screening Committee. Furthermore, the change in cost-impact would have to be calculated for each municipality separately, as they were offering various screening methods and the participation rates varied markedly between the municipalities. Only crude estimates could be given but they were regarded as sufficient to proceed with the statute.

The Ministry of Health and Social Affairs decided to give a new umbrella statute on all screening programs. The official screening programs-mammography screening for women 50 to 69 years of age, screening for cervical cancer, and prenatal screening for structural and chromosomal abnormalities - are now included in one statute, approved by the Council of State on 21 December 21 2006. The uniform screening for structural and chromosomal abnormalities must be adopted in all municipalities by the end of 2010 at the latest.

The explanatory memorandum of the statute sets the criteria for the evaluation of all screening programs that have to be fulfilled before any program can be offered by public health care. These criteria were originally formed by the national Screening Committee (3). Furthermore, the quality and possible harms of any screening program have to be continuously monitored.

\section{THE CONTENT OF THE NATIONAL PRENATAL SCREENING PROGRAM FOR STRUCTURAL AND CHROMOSOMAL ABNORMALITIES}

The content of the statute for the prenatal screening program is explained in depth in the explanatory memorandum. The main content of the statute is as follows: (i) General ultrasound during gestation week 10-14 to provide information on length of gestation, plurality, site of fetus. (ii) Screening for chromosomal abnormalities primarily by a combination test: serum markers PAPP-A and $\beta$-HCG at $8-11$ gestation weeks and nuchal translucency measurement in gestation weeks 10 to 12 in connection with a general ultrasound scan. Alternatively, the triple test (serum markers AFP, $\beta$-HCG, estriol) on gestation weeks 14 to 15 . Women 40 years of age or older can be offered direct fetal chromosomal analysis (placental biopsy or amniocentesis) as an option. Nuchal translucency measurements have to be centralized so that the sonographer performs at least 200 measurements per year. (iii) Screening for structural abnormalities is offered during gestation weeks 18 to 21 if the parents consider termination of pregnancy due to identified severe fetal malformation as an option for their family. If the parents would not consider termination of pregnancy as their informed choice under any circumstances, the scan can be made first after the 24th gestation week. The ultrasound screening for structural abnor- malities have to be centralized so that the unit performs at least 2000 screening scans per year and has the possibility to consult an obstetrician specialized in ultrasound investigations. The time frames and various options are summarized in Figure 1.

\section{DISCUSSION}

Variation in prenatal screening practices in Finnish municipalities had resulted in inequity in care. To solve this problem, an HTA project was launched to help decision makers in choosing best possible screening programs. The assessment was accomplished, but it identified major policy questions that needed public discussion before deciding on how to proceed. The new national Screening Committee opened up the discussion, actively involving health decision makers, professionals, representatives of patient organizations, and the general public

The National Screening Committee compared the different screening options against no publicly funded screening for fetal abnormalities. The need to ensure equity in care was a clear priority. The statement by the Screening committee required much background work, interchange of opinions and weighing the consequences of various value-laden options. We participated both in the HTA project and were members of the Screening Committee so we could provide detailed information at the point when the Committee weighed the different scenarios and screening options. This close cooperation was essential for the content of the final statement.

The National Screening Committee also discussed what could be regarded as an acceptable false positive rate in chromosomal screening as the invasive diagnostic procedures can lead to miscarriage. The rate of miscarriage is around 1 percent (12), being slightly higher after chorionic villi sample than after amniocentesis, and in addition to which the rate of complications is dependent on the performer's experience (4). The national screening committee suggested that the specificity of the method should be set at 97 percent for the combination test and 95 percent for the triple test. The final levels for sensitivity and specificity must be defined by a national group of experts that will start their work autumn 2007.

Neither Finohta, the National Screening Committee, nor the Ministry had originally planned for a new statute on screening. The wide acceptance of the statement on prenatal screening, the strong request to regulate this screening program by means of a statute, and the need to respect the rights of all pregnant women eventually led to the decision that a statute is the only possibility to ensure a uniform and justified screening program.

\section{CONCLUSION}

The HTA project on evaluating the various screening program for fetal chromosomal and structural abnormalities reached 
beyond its original task. Questioning the values of these screening programs led to a nationally uniform screening program in which the objectives and ethical values of various families have been taken into account. In addition, the process supported the formation of an umbrella statute on screening.

\section{CONTACT INFORMATION}

Ilona Autti-Rämö, MD, PhD (ilona.autti-ramo@stakes.fi), Senior Medical Officer, Finnish Office for Health Care Technology Assessment (Finohta), STAKES, Lintulahdenkuja 4, 00530 Helsinki, Finland; Adjunct Professor, Pediatric Neurology, University of Helsinki, Lastenlinnantie 2, 00250 Helsinki, Finland

Marjukka Mäkelä, MD, PhD, MSc (marjukka.makela@ stakes.fi), Director, Finnish Office for Health Care Technology Assessment (Finohta), STAKES (National R\&D Centre for Welfare and Health), P.O. Box 220, 00531 Helsinki, Finland; Professor, Department of General Practice, University of Copenhagen, P.O. Box 2099, 1014 Copenhagen K, Denmark

\section{REFERENCES}

1. Autti-Rämö I, Koskinen H, Mäkelä M, Ritvanen A, and working group. Maternal ultrasound and serum screening in the detection structural and chromosomal abnormalities (in Finnish). FinOHTA report 27. 2006. Available at: http://finohta.stakes.fi.

2. Autti-Rämö I, Mäkelä M. Ethical evaluation in health technology assessment reports. An eclectic approach. Int J Technol Assess Health Care. 2007;23:1-8.
3. Autti-Rämö I, Mäkelä M, Sintonen H, et al. Expanding screening for rare metabolic disease in the newborn: An analysis of costs, effect and ethical consequences for decision making in Finland. Acta Paediatr. 2005;94:1126-1136.

4. Bettelheim D, Kolinek B, Schaller A, Bernaschek G. Complication rates of invasive intrauterine procedures in a centre for prenatal diagnosis and therapy (In German). Ultraschall Med. 2002;23:119-122.

5. Bricker L, Garcia J, Henderson J, et al. Ultrasound screening in pregnancy: A systematic review of the clinical effectiveness, cost-effectiveness and women's views. Health Technol Assess. 2000;4:1-193.

6. Connor JM, Ferguson-Smith MA. Essential medical genetics. 4th ed. Cambridge: The University Press; 1995.

7. Cuckle HS, Wald NJ, Lindenbaum RH. Maternal serum alphafetoprotein measurement: A screening test for Down syndrome. Lancet. 1984;1:926-929.

8. Grandjean H, Larroque D, Levi S. Sensitivity of routine ultrasound screening of pregnancies in the Eurofetus database. The Eurofetus Team. Ann N Y Acad Sci. 1998;847:118-124.

9. Grandjean H, Larroque D, Levi S. The performance of routine ultrasound screening in the Eurofetus database. Am J Obstet Gynecol. 1999;181:446-454.

10. Ritchie K, Boynton J, Bradbury I, et al. Routine ultrasound scanning before 24 week of pregnancy. Report No. 5. Glasgow: NHS Quality Improvement Scotland; 2004.

11. Terho A. Quality criteria and regional differences in trisomy screening in Finland. (In Finnish). Oulu: Oulun Yliopisto; 2002.

12. Wald NJ, Rodeck C, Hacshaw AK, Walters J, Chitty L, Mackinson A. First and second trimester antenatal screening for Down's syndrome: The results of the Serum, Urine and U1trasound Screening Study (SURUSS). Health Technol Assess. 2003; 7:1-88. 